\title{
Fingerprint Pattern of Matching Family with GLCM Feature
}

\author{
Bahtiar Imran*, Karya Gunawan, Muhammad Zohri, Lalu Darmawan Bakti \\ Collage Computer Information Management (STMIK Mataram), Indonesia \\ ${ }^{*}$ Corresponding author, e-mail: bahtiarimranlombok@gmail.com
}

\begin{abstract}
In this research, fingerprint pattern matching is done to find out whether there is the similarity between parent and child fingerprint pattern. An important step in fingerprint matching is the fingerprint pattern search and matching. Fingerprint data is used by 11 families from various families. The method used in fingerprint feature extraction is GLCM. The GLCM angle used is $0^{\circ}$, and the features used are contrast, homogeneity, correlation, and energy. For fingerprint pattern matching use minutiae score. From the results obtained GLCM has been widely used in fingerprint texture analysis. This study proves that the proposed method for matching fingerprints on parents and children gets the most dominant pattern is the loop pattern.
\end{abstract}

Keywords: Fingerprint, GLCM, Pattern, Texture, Analysis

Copyright $\odot 2018$ Universitas Ahmad Dahlan. All rights reserved.

\section{Introduction}

Fingerprint is categorized as one of the best identification tools for its uniqueness [1-2]. Many researchers about combination development [3], feature extraction is well done [4]. Fingerprint matching is an essential and difficult predicament in fingerprint recognition. Still, even if so many different methods are there, it has been erudite from studies that a improved feature extraction technique may leads to especially good outcome [5]. Even though so many different methods are there, it has been learned from studies that a better feature extraction technique may leads to very good results. Co-occurrence matrices can be used to extract features from the fingerprint image because they are composed of regular texture patterns [6].

Fingerprints are the most widely used parameter for personal identification amongst all biometrics. Fingerprint identification is commonly employed in forensic science to aid criminal investigations etc. A fingerprint is a unique pattern of ridges and valleys on the surface of a finger of an individual [7]. In addition, the fingerprint is used for genetics [8], the relationship between the child's fingerprint has a correlation relationship with the parent's fingerprint [9]. Parents with a particular fingerprint pattern have a relative high tendency to produce children with the same pattern [10].

Several previous studies have examined fingerprint matching using the GLCM method. As for the concentration conducted in this study is, fingerprint matching is done on family fingerprint between parents and children. The data used in this study were 11 different family fingerprints. The GLCM method is used to extract parent and child fingerprints. Minutiae score is done to match the fingerprint pattern.

\section{Related Work}

Fingerprint matching is performed based on finding the normalized Euclidean distance between the input and the template feature vectors. Experimental results validate the effectiveness of the proposed method in extracting fingerprint features and achieving good performance [6].

This work uses vector which is generated from Huffman coding compression process. Therefore, the matching process is done between code (vector) and codes (vectors) and the database is sharply decreased. The obtained results are considerably promising since very low FAR i.e. $0.733 \%$, FRR i.e. $2.6 \%$ and high accuracy i.e. approximately $97 \%$ [11]. Finger print recognition and matching algorithm is explained and results give remarkable performance. 
Images are cropped and features are extracted, then matching is done using Euclidean distance [5].

\section{Research Method}

In this study, we determined the classification of the fingerprints of parents and children in accordance with Reviews their respective families. Fingerprint Data taken using a digital fingerprint persona $u$ are $u 4500$ SDK. Then the application used is C\# based applications. Before the stage classification will be done first pre-processing of the fingerprint of data, the size of the original fingerprint $254 \times 292$.At the stage of pre-processing will be Carried outcropping of the fingerprint of data to the size of $154 \times 192$. After the pre-processing stage will be Carried out later on fingerprint feature extraction parents and children to get its value, then it will be done after the pattern matching. The method of feature extraction proposed in this research is gray level co-occurrence matrix (GLCM) method. GLCM features used in this research are correlation, contrast, homogeneity, and energy.

$$
\text { Correlation } \quad f 1=\frac{\sum_{i} \Sigma_{j}\left(i-\mu_{x}\right) \cdot\left(j-\mu_{y}\right) \cdot p(i, j)}{\sqrt{\sigma_{x \sigma y}}}
$$

Where:

$$
\begin{aligned}
& i=\text { row } \\
& j=\text { columns } \\
& p=\text { glcm matrix }
\end{aligned}
$$

$$
\text { Contrast } \quad f 2=\sum_{i} \sum_{j}(i-j)^{2} \cdot p(i, j)
$$

Measuring the spatial frequency of the image and the difference GLCM moment. The difference is meant a difference of its high and low pixel. Contrast will be 0 if the pixel neighborhoods have the same value.

$$
\text { Homogeneity } \quad f 3=\sum_{i} \sum_{j} \frac{\mathbf{p}(i, j)}{1+(i-j)^{2}}
$$

The value was very sensitive to the value around the main diagonal. It will has a high value when all the pixels have the same value. The opposite of contrast will have a great value if it has the same pixel value at the time of valuable energy.

$$
\text { Energy } \quad f 4=\sum_{i} \sum_{j} p(i-j)^{2}
$$

Energy will have a high value when the pixel values are similar to one another would otherwise be of little value indicates the value of GLCM normalization is heterogeneous. The maximum value of the energy was 1 , which means the distribution of pixels in a state of constant or in the form (not random). The main steps of proposed method are:

1. Data collection fingerprint

2. Image resampling to $254 \times 292$ number of pixel size.

3. Pre-processing the input image (154 x 192).

4. Region-of-interest

5. Feature extraction GLCM

6. Determination of fingerprint pattern using minutiae score.

\section{Results and Analysis}

\subsection{Data Collection Fingerprint}

Data were taken from 11 different families in the Lombok region. The fingerprints were taken our children's fingerprints and parental fingerprints. The total fingerprint used in this study was 33 fingerprints. 


\subsection{Image Resampling} in Figure 1.

In this study, using the original fingerprint image with a size of $254 \times 292$ pixels as shown
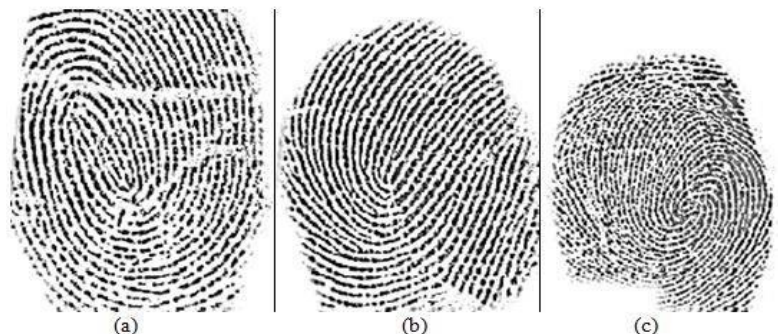

Figure 1. Fingerprint original (a) father (b) mother (c) child

\subsection{Preprocessing}

In the preprocessing stage will be cropping on the original fingerprint image into a $154 \times 192$ pixels to reduce the computing process, $154 \times 192$ cropping results can be seen in Figure 2.

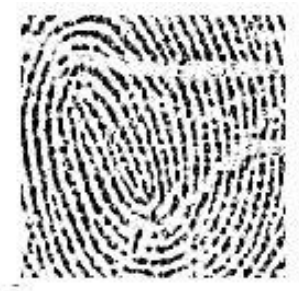

(a)

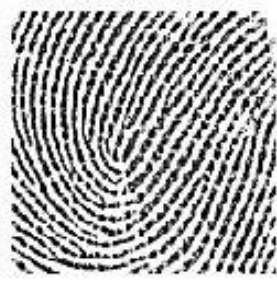

(b)

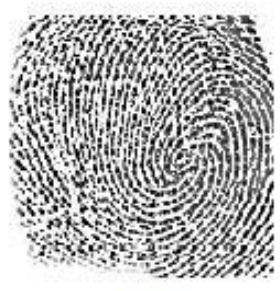

(c)

Figure 2. Cropping fingerprint (a) father (b) mother (c) child

\subsection{Region of Interest}

At this stage we will look for the ROI of fingerprints father, mother and children to speed up the process of classification, the results of fingerprint $\mathrm{ROI}$ as Figure 3.

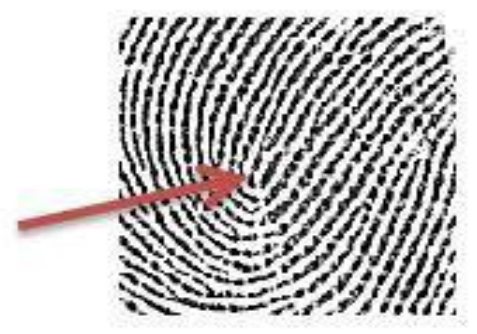

Figure 3. Region of interest

\subsection{Feature Extraction GLCM}

Feature extraction to obtain fingerprint features, parent, and child. Angles used are $0^{\circ}$ and features used are energy, contrast, correlation, and homogeneity. The results of fingerprint feature extraction for parents and children such as in Table 1. 
Table 1. GLCM

\begin{tabular}{llllll}
\hline No & Name & Contrast & Homogenity & Correlation & Energy \\
\hline Family 1 & Father & 9347.3 & 0.3497 & 0.5049 & 0.0978 \\
& Mother & 8402.3 & 0.4321 & 0.5654 & 0.1470 \\
Family 2 & Child & 6903.3 & 0.5202 & 0.5558 & 0.2478 \\
& Father & 8160.2 & 0.4209 & 0.5438 & 0.1447 \\
& Mother & 8402.3 & 0.4321 & 0.5654 & 0.1470 \\
Family 3 & Child & 9723.9 & 0.4101 & 0.4918 & 0.1290 \\
& Father & 8160.2 & 0.4209 & 0.5438 & 0.1447 \\
& Mother & 8402.3 & 0.4321 & 0.5654 & 0.1470 \\
Family 4 & Child & 9181.6 & 0.4057 & 0.4507 & 0.1353 \\
& Father & 8498.5 & 0.4670 & 0.5289 & 0.1748 \\
& Mother & 9611.9 & 0.4768 & 0.4967 & 0.1921 \\
Family 5 & Child & 7957.9 & 0.5112 & 0.4334 & 0.2466 \\
& Father & 7525.4 & 0.4553 & 0.5487 & 0.1678 \\
& Mother & 6659.4 & 0.3669 & 0.5919 & 0.1864 \\
Family 6 6 & Child & 6530.0 & 0.5060 & 0.4868 & 0.2437 \\
& Father & 8751.3 & 0.3846 & 0.4845 & 0.1183 \\
& Mother & 10448 & 0.3229 & 0.4082 & 0.0840 \\
Family 7 & Child & 9710.9 & 0.3355 & 0.4619 & 0.0924 \\
& Father & 8751.3 & 0.3846 & 0.4845 & 0.1183 \\
& Mother & 10448 & 0.3229 & 0.4082 & 0.0840 \\
Family 8 & Child & 9034.0 & 0.3607 & 0.4371 & 0.1048 \\
& Father & 8751.3 & 0.3846 & 0.4845 & 0.1183 \\
& Mother & 10448 & 0.3229 & 0.4082 & 0.0840 \\
Family 9 9 & Child & 7490.3 & 0.4672 & 0.5134 & 0.1905 \\
& Father & 7313.2 & 0.3799 & 0.6170 & 0.1137 \\
& Mother & 8470.9 & 0.4875 & 0.4898 & 0.2128 \\
& Child & 8254.4 & 0.4921 & 0.5397 & 0.2132 \\
& Father & 9801.7 & 0.3452 & 0.4565 & 0.0967 \\
& Mother & 9053.9 & 0.3411 & 0.5282 & 0.0909 \\
& Child & 12617 & 0.3764 & 0.3793 & 0.1144 \\
& Father & 10374 & 0.3227 & 0.4117 & 0.1518 \\
& Mother & 7852.2 & 0.4341 & 0.5754 & 0.1691 \\
\hline & Child & 5918.9 & 0.4604 & 0.6899 &
\end{tabular}

\subsection{Fingerprint Pattern}

Pattern matching to facilitate the classification of each fingerprint pattern after pattern recognition is done according to each family. Fingerprint matching is done using minutiae score by counting the minutiae detected later in comparison with minutiae on other fingerprints. Example minutiae detected in Figure 4, to view the results of Table 2.

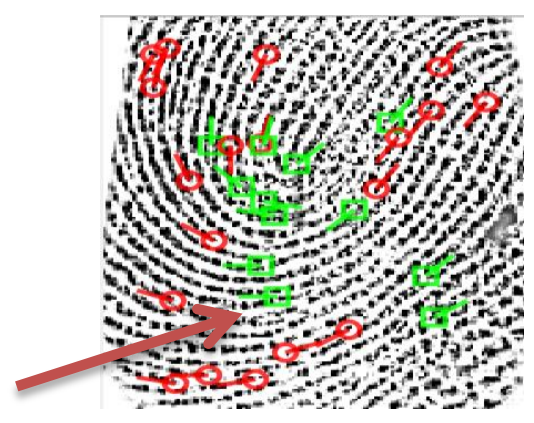

Figure 4. Minutiae detection

This study uses 11 family fingerprint data consisting of father's fingerprint, mother's fingerprint, and child's fingerprint, the total number of fingerprints used is 33 fingerprints. Gray level co-occurrence matrix (GLCM) method is used to obtain the extraction result on fingerprint, 
the angle used in this research is angle $0^{\circ}$. Minutiae score is used to calculate the number of detected minutiae so that it can be matched with every minutia of the father's fingerprint, mother's fingerprint, and child's fingerprint.

Table 2. GLCM and Fingerprint pattern using minutiae score

\begin{tabular}{|c|c|c|c|c|c|c|c|}
\hline No & Name & Contrast & Homogenity & Correlation & Energy & Minutiae & Pattern \\
\hline \multirow[t]{3}{*}{ Family 1} & Father & 9347.3 & 0.3497 & 0.5049 & 0.0978 & 30 & Loop \\
\hline & Mother & 8402.3 & 0.4321 & 0.5654 & 0.1470 & 32 & Loop \\
\hline & Child & 6903.3 & 0.5202 & 0.5558 & 0.2478 & 30 & Loop \\
\hline \multirow[t]{3}{*}{ Family 2} & Father & 8160.2 & 0.4209 & 0.5438 & 0.1447 & 35 & Loop \\
\hline & Mother & 8402.3 & 0.4321 & 0.5654 & 0.1470 & 32 & Loop \\
\hline & Child & 9723.9 & 0.4101 & 0.4918 & 0.1290 & 36 & Loop \\
\hline \multirow[t]{3}{*}{ Family 3} & Father & 8160.2 & 0.4209 & 0.5438 & 0.1447 & 35 & Loop \\
\hline & Mother & 8402.3 & 0.4321 & 0.5654 & 0.1470 & 32 & Loop \\
\hline & Child & 9181.6 & 0.4057 & 0.4507 & 0.1353 & 39 & Loop \\
\hline \multirow[t]{3}{*}{ Family 4} & Father & 8498.5 & 0.4670 & 0.5289 & 0.1748 & 31 & Loop \\
\hline & Mother & 9611.9 & 0.4768 & 0.4967 & 0.1921 & 33 & Loop \\
\hline & Child & 7957.9 & 0.5112 & 0.4334 & 0.2466 & 17 & Whorl \\
\hline \multirow[t]{3}{*}{ Family 5} & Father & 7525.4 & 0.4553 & 0.5487 & 0.1678 & 28 & Whorl \\
\hline & Mother & 6659.4 & 0.3669 & 0.5919 & 0.1864 & 35 & Loop \\
\hline & Child & 6530.0 & 0.5060 & 0.4868 & 0.2437 & 20 & Whorl \\
\hline \multirow[t]{3}{*}{ Family 6} & Father & 8751.3 & 0.3846 & 0.4845 & 0.1183 & 32 & Loop \\
\hline & Mother & 10448 & 0.3229 & 0.4082 & 0.0840 & 39 & Loop \\
\hline & Child & 9710.9 & 0.3355 & 0.4619 & 0.0924 & 38 & Loop \\
\hline \multirow[t]{3}{*}{ Family 7} & Father & 8751.3 & 0.3846 & 0.4845 & 0.1183 & 32 & Loop \\
\hline & Mother & 10448 & 0.3229 & 0.4082 & 0.0840 & 39 & Loop \\
\hline & Child & 9034.0 & 0.3607 & 0.4371 & 0.1048 & 45 & Whorl \\
\hline \multirow[t]{3}{*}{ Family 8} & Father & 8751.3 & 0.3846 & 0.4845 & 0.1183 & 32 & Loop \\
\hline & Mother & 10448 & 0.3229 & 0.4082 & 0.0840 & 39 & Loop \\
\hline & Child & 7490.3 & 0.4672 & 0.5134 & 0.1905 & 53 & Whorl \\
\hline \multirow[t]{3}{*}{ Family 9} & Father & 7313.2 & 0.3799 & 0.6170 & 0.1137 & 31 & Loop \\
\hline & Mother & 8470.9 & 0.4875 & 0.4898 & 0.2128 & 30 & Loop \\
\hline & Child & 8254.4 & 0.4921 & 0.5397 & 0.2132 & 39 & Loop \\
\hline \multirow[t]{3}{*}{ Family 10} & Father & 9801.7 & 0.3452 & 0.4565 & 0.0967 & 52 & Loop \\
\hline & Mother & 9053.9 & 0.3411 & 0.5282 & 0.0909 & 51 & Loop \\
\hline & Child & 12617 & 0.3764 & 0.3793 & 0.1144 & 52 & Loop \\
\hline \multirow[t]{3}{*}{ Family 11} & Father & 10374 & 0.3227 & 0.4117 & 0.0829 & 41 & Loop \\
\hline & Mother & 7852.2 & 0.4341 & 0.5754 & 0.1518 & 41 & Loop \\
\hline & Child & 5918.9 & 0.4604 & 0.6899 & 0.1691 & 42 & Loop \\
\hline
\end{tabular}

\section{Conclusion}

In this study presents two methods of fingerprint classification of parents and children. There are two major contributions to research. The first is fingerprint extraction using GLCM to get the value of a feature. The second is the use of the minutiae score method for matching fingerprint patterns. GLCM is also used to perform fingerprint analysis. The data used are 11 family fingerprints consisting of child fingerprint, father's fingerprint, mother's fingerprint. The most dominant pattern is the loop pattern. The success rate of the minutiae extraction process depends heavily on the quality of the fingerprint image. Image obtained with low image quality can result in minutiae process is not maximal so minutiae cannot be found.

\section{References}

[1] Soman MA, Avadhani R, Jacob M, Nallathamby R. Study of Fingerprint Patterns in Relationship with Blood group and Gender. Research Journal of Forensic Sciences. 2013; 1(1): 15-17.

[2] Chen W, Gao Y. A Minutiae-based Fingerprint Matching Algorithm Using Phase Correlation. Digital Image Computing Techniques and Applications. 2007; 233-238.

[3] Mohaniah P, Sathyanarayana, Guru Kumar L. Image Texture Feature Extraction Using GLCM Approach. International Journal of Scientific and Research Publications. 2013; 3(5): 1-5. 
[4] Pathak B, Barooah D. Texture Analysis Based on the Gray-Level Co-occurrence Matrix Considering Possible Orientations. International Journal of Advanced Research in Electrical, Electronics and Instrumentation Engineering. 2013; 2(9): 4206-4212.

[5] Mishra A, Maheshwari P. An Efficient System for Fingerprint Finger Print Matching and Classification. International Journal of Advanced Research in Computer Science and Software Engineering. 2013; 3(11): 295-300.

[6] KKB Vijayakumar. Fingerprint Matching by Extracting GLCM Features. International Conference \& Workshop on Recent Trends in Technology Proceedings published in International Journal of Computer Applications ${ }^{\circledR}$ (IJCA), (TCET). 2012: 30-34

[7] Verma P, Bahendwar Y, Sahu A, Dubey A. Feature Extraction Algorithm of Fingerprint Recognition. International Journal of Advanced Research in Computer Science and Software Engineering. 2012; 2(10): 292-297.

[8] Abraham AT, MYK. Genotyping-Wavelet Approach. International Journal of Science and Research (IJSR). 2013; 4(2): 1025-1027.

[9] Suharjito, Imran B, Girsang A S. Family Relationship Identification by Using Extract Feature of Gray Level Co-occurrence Matrix (GLCM) Based on Parents and Children Fingerprint. International Journal of Electrical and Computer Engineering (IJECE). 2017; 7(5): 2738-2745.

[10] Matsuyama N, Ito Y. The Frequency of Fingerprint Type in Parents of Children with Trisomy 21 in Japan. Journal of Physiological Anthropology. 2006: 15-21.

[11] Aburas AA, Rahiel SA. Fingerprint Patterns Recognition System Using Huffman Coding. Proceedings of the World Congress on Engineering. London, U.K. 2008; III. 